\title{
The relevance of the pretreatment on the chemical modification of cellulosic fibers
}

\author{
Roberto Aguado (id - Ana F. Lourenço • Paulo J. T. Ferreira • Ana Moral • \\ Antonio Tijero
}

Received: 28 January 2019/Accepted: 15 May 2019

(C) Springer Nature B.V. 2019

\begin{abstract}
Cationized fibers and other kinds of chemically modified fibers impart many advantages in papermaking, but unfeasibly long reaction times are necessary to attain acceptable degrees of substitution, due to the low reactivity of bleached kraft pulps. In this work, different aqueous pretreatments were tested in order to activate cellulose towards a 60 min-long etherification with a quaternary ammonium reagent. Severe decrystallization treatments, namely alkalization with $\mathrm{NaOH} 20 \%, \mathrm{NaOH} /$ urea or FeTNa, conducted to the best reactivity results (substitution from 2 to $10 \%$ ), but the fiber properties were harshly
\end{abstract}

affected. Pretreatments involving $\mathrm{H}_{3} \mathrm{PO}_{4}$ at different concentrations were also performed, with distinct results, from unnoticeable effects at $20 \%$ to amorphization and excessive depolymerization at $80 \%$. Finally, aqueous ammonium thiocyanate was tested as activator and had little effect on fibers, although the addition of ammonia resulted in high degrees of substitution, while maintaining the pulps' capability to retain inter-fiber water and cellulose I as the prevalent allomorph.

Electronic supplementary material The online version of this article (https://doi.org/10.1007/s10570-019-02517-7) contains supplementary material, which is available to authorized users.

R. Aguado $(\bowtie) \cdot$ A. Moral

Molecular Biology and Biochemical Engineering

Department, ECOWAL, Pablo de Olavide University,

41013 Seville, Spain

e-mail: rjagugar@alumno.upo.es

A. F. Lourenço · P. J. T. Ferreira

Chemical Engineering Department, CIEPQPF, University

of Coimbra, 3030-790 Coimbra, Portugal

\author{
A. Tijero \\ Grupo de Celulosa y Papel, Chemical Engineering \\ Department, Complutense University of Madrid, \\ 28040 Madrid, Spain
}




\section{Graphical abstract}

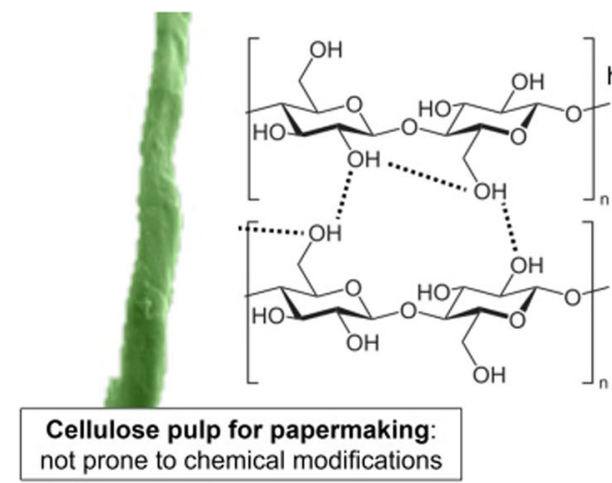

Keywords Activation - Cationization of cellulose · Degree of crystallinity · Pretreatment · Swelling .

$\mathrm{X}$-ray diffraction

\section{Introduction}

Despite the usefulness of many cellulose derivatives, the conditions required currently by heterogeneous etherifications make a fiber functionalization stage impractical in a pulp or paper mill. Modified starches are widely used as wet-end additives, but fibers are always essentially composed of native cellulose, even though they could also attain interesting functionalities. For instance, inserting cationic functional groups, commonly quaternary ammonium, into the structure of cellulose fibers and/or fines can compensate their negative charge density (Sood et al. 2010), improve the internal bond strength of paper (Montplaisir et al. 2006), diminish the dependence on poorly biodegradable polymeric aids (Aguado et al. 2017), and decrease drainage time during sheet forming (Mayr et al. 2017). Sehaqui et al. (2016) produced cationic cellulose nanofibers with quaternary ammonium groups, and proved their usefulness in water treatment processes, as they could retain fluoride, nitrate, and other anions. Antimicrobial properties have also been shown, with death rates of $98 \%$ or even higher for Candida oleophila (Littunen et al. 2016). This chemical reaction, commonly known as cationization, is an etherification of some hydroxyl groups of cellulose in, most frequently, an aqueous alkaline medium (Prado and Matulewicz 2014).
The biggest hindrance to be overcome is the high crystallinity and thermodynamic stability of cellulose in pulps for papermaking. Mayr et al. (2017) obtained a degree of substitution of only 0.034 after treating fines with a quaternary ammonium epoxide for $15 \mathrm{~h}$. Most likely, the best conversion values result from oxidation followed by Girard's reagent T (Sirviö et al. 2014), but etherifications are still more common in the literature, also more common than crosslinking (Gruber et al. 1996). Furthermore, our work aims to study the effects of pretreatments not only for cationization, but also for a broad variety of chemical modifications on cellulose. Previous kinetic studies of cellulose etherification have suggested that the reaction takes place quickly in activated regions of fibers, but very slowly in stable regions (Hedlund and Germgård 2007). In a recent article, the authors have proved that the cationization of cellulose fibers showed similar kinetics (Aguado et al. 2018), as the degree of substitution stabilized after a certain time, and further functionalization could be obtained if prior activation of fibers was performed.

Cellulose polymorphism is due to the intramolecular and intermolecular hydrogen bonding system. Cellulose in fibers from wood and plants is essentially cellulose I $\beta$, a form with a certain crystal structure that was described in detail by Nishiyama et al. (2002). Some treatments may affect hydrogen bonding between hydroxyl groups, thus decreasing crystallinity or converting an allomorph into another. Decrystallization, i.e., the creation of amorphous domains, is one of the ways of activating cellulose for functionalization, since it increases the entropy of the polymer. Other ways involve swelling and 
ionization. Alternatively, if the reaction desired is especially difficult, an easier nucleophilic substitution can be performed beforehand, as it is easier to transform already modified cellulose (Heinze 2004).

Although certain organic polar liquids and molten salts can be used to activate cellulose (Fischer 2004), this work focuses on aqueous pretreatments, as the subsequent functionalization is carried out in an aqueous solution. In order to obtain cationic fibers with high strength, the cellulose activator must swell the fibers, but not dissolve them. Generally speaking, cellulose solvents can be used as activators if the conditions are milder than those used for dissolution. In fact, some sort of activation is always the first stage of dissolution in aqueous media (Zhang et al. 2009).

In this paper, the objective is to compare four different activating pretreatments under a total of 26 sets of experimental conditions, in order to test their influence on cellulose fibers and to improve the results of the following reaction. Then, cationization is carried out under mild conditions. Despite keeping the focus on cationization, the findings could be extrapolated to other etherifications of polysaccharides. The selection of cellulose activators include sodium hydroxide, more widely used than any other in previous studies, but also some agents used in the literature to dissolve this polymer: orthophosphoric acid $\left(\mathrm{H}_{3} \mathrm{PO}_{4}\right)$ (Vinogradov et al. 2002), FeTNa (Vu Mahn et al. 2010), ammonium thiocyanate $\left(\mathrm{NH}_{4} \mathrm{SCN}\right)$ with or without ammonia (Mahmud-Ali and Bechtold 2015), and several combinations with urea (Jin et al. 2007). It is hypothesized that chemical modifications of wood pulp by etherification can yield satisfactory results with a short reaction time, as long as a pretreatment that produces the proper structural changes in cellulose has been applied.

\section{Materials and methods}

Pulp and reagents

All pretreatments and reactions took place on a bleached kraft pulp from Eucalyptus globulus (BEKP), also used in a previous work of ours (Aguado et al. 2017). The Schopper-Riegler freeness was 18 ${ }^{\circ} \mathrm{SR}$. Its composition, determined by following the common TAPPI methods, resulted in $82 \% \alpha$-cellulose (T429 cm-10), 0.4\% ashes (T211 om-02), 0\% acid- insoluble lignin (T122 om-15), kappa number of 1 (T236 om-99). The average molecular weight (M, Da) of the cellulose pulp, estimated from the limiting viscosity number $(\eta, \mathrm{mL} / \mathrm{g})$ measured in a copper(II) ethylenediamine solution (Cuen) as defined in ISO 5351, by applying the Mark-Howink equation (Eq. 1) with the parameters for cellulose reported by Eckelt et al. (2011), was found to be $189 \mathrm{kDa}$.

$\eta=0.0653 \mathrm{M}^{0.735}$

An aqueous solution of orthophosphoric acid $\left(\mathrm{H}_{3} \mathrm{PO}_{4}, 85 \%\right)$, urea pearls, sodium tartrate dihydrate and iron trichloride $\left(\mathrm{FeCl}_{3}\right)$ were purchased from Panreac (Barcelona). A solution of (3-chloro-2-hydroxypropyl)trimethylammonium chloride (CHPTAC) with a mass concentration of $60 \%$ was obtained from SigmaAldrich (Madrid).

BEKP fibers were disintegrated in water at $3000 \mathrm{rpm}$ (disintegrator from Lorentzen and Wettre), in accordance to ISO 5263. The consistency was adjusted to $10 \%$ and the pulp was stored at temperatures below $10{ }^{\circ} \mathrm{C}$.

To synthetize FeT, an iron(III)-tartrate complex, $20 \mathrm{~g}$ of $\mathrm{FeCl}_{3}$ and $96 \mathrm{~g}$ of sodium tartrate dihydrate were dissolved in $200 \mathrm{~mL}$ of distilled water, adding sulphuric acid to adjust the $\mathrm{pH}$ to 4 . The dissolution was submitted to gentle stirring (200 rpm) for $30 \mathrm{~min}$. By pouring it into $300 \mathrm{~mL}$ of acetone, the coordination compound immediately precipitated as a yellow powder. The solid and the liquid were separated by filtration. FeT became FeTNa in a $\mathrm{NaOH}$ solution (ElWakil and Hassan 2008).

The experimental procedure, including the characterization of both the pretreated fibers and the functionalized fibers, is schematized in Fig. 1.

\section{Pretreatments}

All the pretreatment processes, which involved the reagents listed in Fig. 1 at different concentrations, took place at constant temperature and with a 3-bladed stirrer $(600 \mathrm{rpm})$, by means of an Agimatic-E plate (Selecta) with a PID device including a Pt-100 probe from Sensotherm. The pulp was soaked in the activation solutions at a liquid-to-solid ratio of 20 .

It should be pointed out that the pretreatments with $\mathrm{NaOH} /$ urea required adding the urea pearls to a precooled $\mathrm{NaOH}$ solution. Otherwise, the exothermic 


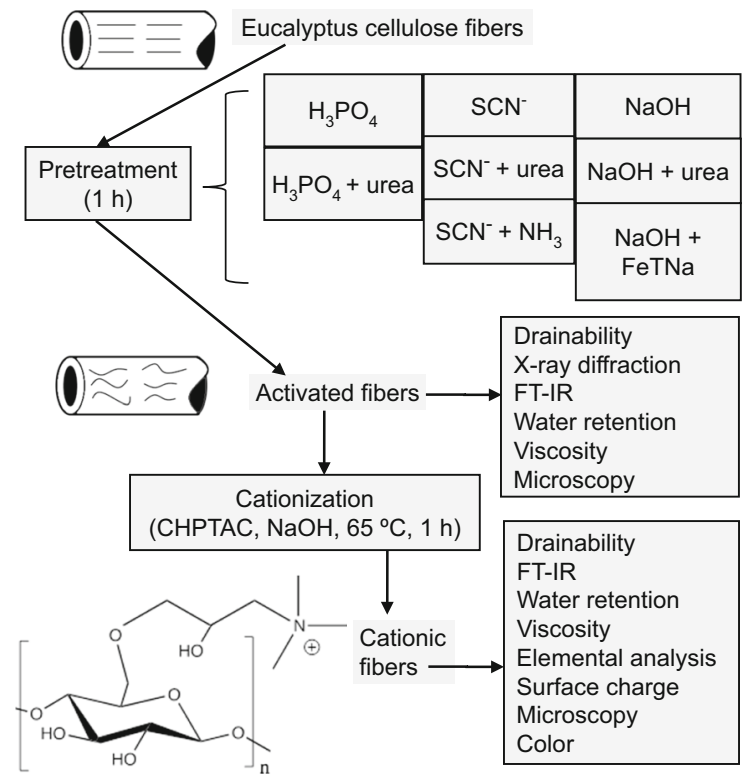

Fig. 1 Simplified diagram of experimentation

dissolution of the alkali would have prompted the hydrolysis of urea. Treatments with $\mathrm{NaOH}$ at $60{ }^{\circ} \mathrm{C}$ were disregarded, since this activation is known to work better at low temperature (Jin et al. 2007).

After 60 min of agitation and heating, the suspensions were diluted in water to a pulp consistency of $1 \%$. Then, they were vacuum-filtered through an $11 \mu \mathrm{m}$ paper filter. The solids were weighted to determine the yield towards non-solubilized fibers. A small portion (less than 2\%) of the solid fraction was used to measure the moisture content by drying at $105^{\circ} \mathrm{C}$ (ISO 638). The filtrate was poured into ethanol, with an ethanol-to-water ratio of 1 , to regenerate dissolved cellulose, if any. The previous solid fraction was also poured into the suspension to ensure that the swelling effects of ethanol were the same for all pretreatments. This suspension was filtered again and left to dry at room temperature for $24 \mathrm{~h}$.

\section{Cationization}

Etherification of cellulose with CHPTAC was carried out under mild conditions. Assuming that BEKP consisted solely of anhydroglucose units (AGU), it was soaked in a CHPTAC/NaOH aqueous solution so that the CHPTAC/AGU mole ratio was 1.6 and the $\mathrm{NaOH} / \mathrm{CHPTAC}$ mole ratio was 1.2.
In all cases, the temperature was kept at $65{ }^{\circ} \mathrm{C}$ for 60 min., with a pulp consistency of $3 \%$. Afterwards, the suspension was neutralized with diluted $\mathrm{HCl}$ and poured into ethanol to regenerate cationic cellulose in solution. Fibers were retained on a Whatman filter $(11 \mu \mathrm{m})$, washed with distilled water, and allowed to reach equilibrium moisture at approximately $23{ }^{\circ} \mathrm{C}$.

The percentage of nitrogen $(\% \mathrm{~N})$ in the cationic fibers was determined by combustion with LECO's elemental analyzer CNS-2000I. The degree of substitution (DS) was estimated from Eq. 2 (Aguado et al. 2018). A Charge Analysis System device from AFG was used to measure the surface charge density (CD). Since the equipment is designed for liquids, a back titration method was used instead of a direct one. For that, a small sample of cationized pulp was soaked in excess of an anionic polyelectrolyte, sodium polyethylenlsulphate (PESNa, $1.8 \mathrm{meq} / \mathrm{L})$. The liquid was titrated with a cationic polyelectrolyte, polydiallyldimethylammonium chloride (PDADMAC, $2.2 \mathrm{meq} / \mathrm{L}$ ). The endpoint of the potentiometric titration was the isoelectric point $(0 \mathrm{mV})$. Inversely, to measure the charge density of BEKP, a sample was soaked in PDADMAC and the titrant was PESNa.

$\mathrm{DS}=162 \% \mathrm{~N} /(1401-152 \% \mathrm{~N})$

\section{Observation of structural changes}

Representative samples of pretreated pulps, once dried, were submitted to a PANalytical's powder Xray diffractometer using X'Pert software. Gaussian deconvolutions of the diffraction patterns and integrations of the peaks were performed with Systat's Peakfit software. The crystallinity index (CI) was calculated as follows:

$$
\begin{aligned}
\mathrm{CI}= & \left(\mathrm{A}_{1-10(\mathrm{I} \beta)}+\mathrm{A}_{110(\mathrm{I} \beta)}+\mathrm{A}_{200(\mathrm{I} \beta)}+\mathrm{A}_{110(\mathrm{II})}\right. \\
& \left.+\mathrm{A}_{020(\mathrm{II})}+\mathrm{A}_{004}\right) / \mathrm{A}_{\text {TOTAL }}
\end{aligned}
$$

In Eq. 3, $A$ is the area under each of the Gaussian peaks resulting from integration. Miller indices 1 $10(I \beta), 110(I \beta), 200(I \beta), 110(I I), 020(I I)$ and 004 correspond to the characteristic peaks of cellulose $\mathrm{I} \beta$, II or, in the case of plane 004, both crystalline allomorphs (French 2014).

The limiting viscosity number $(\eta)$ was measured for both the pretreated and the cationized pulps. Pulp viscosity was measured by following the ISO standard 
5351. Briefly, a sample was completely dissolved in Cuen and the time taken to flow through a CannonFenske capillary viscometer was compared to that of the blank solution. Then, Eq. 1 was used to estimate the average molecular weight, which was divided by the molecular weight of AGU, 162, to calculate the degree of polymerization (DP) of the pretreated samples.

Certain pretreated and cationic samples were chosen, along with BEKP, to obtain ATR-FTIR spectra by using a Bruker Tensor 27 spectrometer. Scans were carried out from 3800 to $800 \mathrm{~cm}^{-1}$, with a resolution of $4 \mathrm{~cm}^{-1}$. Furthermore, some visible light absorption spectra were produced by means of a Varian Cary 50 UV-Vis spectrophotometer, from 400 to $750 \mathrm{~nm}$.

\section{Analysis of fiber properties}

The Schopper-Riegler freeness was measured in a DFR-05 device from Mütek, by using a 70 mesh screen $(212 \mu \mathrm{m}$ of aperture size $)$. The measurement was performed in a suspension of $2 \mathrm{~g}$ of pretreated pulp in $1 \mathrm{~L}$ of demineralized water with the "Freeness" settings.

The pulps were also observed by scanning electron microscopy (SEM from JEOL, model JM-6400). Vacuum-dried fibers were distributed on a cylindrical slide, which was coated with gold.

Water retention value (WRV) was determined in accordance with the SCAN-C method 62:00, as the ratio $(\mathrm{w} / \mathrm{w})$ of water retained by a test pad of fibers after centrifugation $(3000 \mathrm{~g}, 15 \mathrm{~min})$ to the dry pad. The test pad was formed by filtration of the samples dilute suspensions on a $100 \mu \mathrm{m}$ filter paper. It must be noted that the WRV calculated by following this method is notoriously lower than that measured by the TAPPI useful method 256 (900 $\mathrm{g}$ for $30 \mathrm{~min}$ ).

\section{Results}

Direct effects of pretreatments

Table 1 shows WRV, the Schopper-Riegler number, the limiting viscosity, the average degree of polymerization and the yield for each set of experimental conditions. Calculations were performed assuming that the pretreatments are not derivatizing. Since water is a swelling agent itself, the original pulp treated with water alone (numbered 0 in Table 1) was submitted to the same mechanical treatments as the rest of the cases. This way, the focus can be put on chemical activation.

By using the pretreatments involving $\mathrm{H}_{3} \mathrm{PO}_{4}$ at high concentrations (Table 1, sample codes 11 and 12), $\mathrm{NaOH} 20 \%$ (code 14), NaOH/urea (codes 15,16 ) or $\mathrm{NH}_{4} \mathrm{SCN} 60 \%$ (code 26), fibers swelled far more than when they were soaked in water alone (code 0 ), as can be observed from the water retention value. However, WRV decreased from 1.6 to $1.1 \mathrm{~g} / \mathrm{g}$ after a treatment with $\mathrm{FeT} 10 \%$ and $\mathrm{NaOH} 6 \%$ (code 20), but it must be taken into account that fibers suffered drastic structural changes. They formed a dark green gel as soon as stirring started and most of them were regenerated upon dilution, but they kept an orange-to-red color after neutralization and rinsing. Supplementary material shows that $\mathrm{Fe}$ atoms were somehow inserted into the structure of cellulose, even when FeT concentration was $2.5 \%$.

WRV indicates the capability of the fibers to retain intra-fiber water while the Schopper-Riegler number (Table 1) shows the capability to retain inter-fiber water when a fast filtration process occurs. Yield values below $100 \%$ may not only indicate the dissolution of cellulose or its hydrolysis towards sugars, but also the dissolution and hydrolysis of hemicellulose, generally less stable. Regarding viscosity, it must be taken into account that the repeatability reported in the corresponding TAPPI standard (T230 om-13) is $4 \%$, and thus, slight differences in this measurement can be simply due to random error.

Figure 2 displays SEM images for the original pulp that had been treated with water alone (Fig. 2a, d and $\mathrm{g}$ ), and for pretreatments that have been numbered in Table 1 as 10 (Fig. 2b), 14 (Fig. 2c), 23 (Fig. 2e), 16 (Fig. 2f), 25 (Fig. 2h) and 18 (Fig. 2i), at different magnification values. Considering that the pulp pad was prepared in the same way for all samples, treatments with $\mathrm{H} 3 \mathrm{PO} 460 \%$ or with $\mathrm{NaOH}$ resulted in less packed density and less ease to form fiber bundles (Fig. 2b, c). In particular, the alkaline treatments resulted in clear external fibrillation (Fig. 2c, f and i).

Partial amorphization of fibers can be estimated from the X-ray diffraction patterns in Fig. 3. The Miller indices for the most notorious crystalline peaks are shown, and the crystallinity index (CI) was 
Table 1 Conditions and general results for the different pretreatments

\begin{tabular}{|c|c|c|c|c|c|c|c|}
\hline Code & Activation agent & $\begin{array}{l}\mathrm{T} \\
\left({ }^{\circ} \mathrm{C}\right)\end{array}$ & $\begin{array}{l}\text { WRV } \\
(\mathrm{g} / \mathrm{g})\end{array}$ & $\begin{array}{l}\text { Schopper-Riegler } \\
\left({ }^{\circ} \mathrm{SR}\right)\end{array}$ & $\eta_{(\mathrm{mL} / \mathrm{g})}$ & $\begin{array}{l}\text { Average } \\
\text { DP }\end{array}$ & $\begin{array}{l}\text { Yield } \\
(\%)\end{array}$ \\
\hline 0 & Water & 23 & 1.6 & 18 & 491 & 1159 & 100 \\
\hline 1 & $\mathrm{H}_{3} \mathrm{PO}_{4}$ (aq.) $20 \% \mathrm{w} / \mathrm{w}$ & 30 & 1.5 & 18 & 478 & 1118 & 100 \\
\hline 2 & $\mathrm{H}_{3} \mathrm{PO}_{4}$ (aq.) $20 \% \mathrm{w} / \mathrm{w}$ & 60 & 1.6 & 18 & 502 & 1195 & 99 \\
\hline 3 & $\mathrm{H}_{3} \mathrm{PO}_{4}$ (aq.) $40 \% \mathrm{w} / \mathrm{w}$ & 30 & 1.7 & 15 & 481 & 1127 & 94 \\
\hline 4 & $\mathrm{H}_{3} \mathrm{PO}_{4}$ (aq.) $40 \% \mathrm{w} / \mathrm{w}$ & 60 & 1.8 & 16 & 482 & 1130 & 90 \\
\hline 5 & $\mathrm{H}_{3} \mathrm{PO}_{4}$ (aq.) $40 \% \mathrm{w} / \mathrm{w}+$ Urea $2.5 \%$ w/w & 30 & 1.8 & 15 & 490 & 1156 & 92 \\
\hline 6 & $\mathrm{H}_{3} \mathrm{PO}_{4}$ (aq.) $40 \% \mathrm{w} / \mathrm{w}+$ Urea $2.5 \% \mathrm{w} / \mathrm{w}$ & 60 & 1.8 & 15 & 461 & 1064 & 90 \\
\hline 7 & $\mathrm{H}_{3} \mathrm{PO}_{4}$ (aq.) $60 \% \mathrm{w} / \mathrm{w}$ & 30 & 1.7 & 13 & 413 & 916 & 84 \\
\hline 8 & $\mathrm{H}_{3} \mathrm{PO}_{4}$ (aq.) $60 \% \mathrm{w} / \mathrm{w}$ & 60 & 1.9 & 12 & 340 & 703 & 82 \\
\hline 9 & $\mathrm{H}_{3} \mathrm{PO}_{4}$ (aq.) $60 \%$ w/w + Urea $2.5 \%$ w/w & 30 & 1.9 & 13 & 405 & 892 & 83 \\
\hline 10 & $\mathrm{H}_{3} \mathrm{PO}_{4}$ (aq.) $60 \% \mathrm{w} / \mathrm{w}+$ Urea $2.5 \%$ w/w & 60 & 2.0 & 12 & 352 & 737 & 80 \\
\hline 11 & $\mathrm{H}_{3} \mathrm{PO}_{4}$ (aq.) $80 \%$ w/w & 30 & 2.4 & $<10$ & 210 & 365 & 73 \\
\hline 12 & $\mathrm{H}_{3} \mathrm{PO}_{4}$ (aq.) $80 \% \mathrm{w} / \mathrm{w}$ & 60 & 2.2 & $<10$ & 147 & 225 & 60 \\
\hline 13 & $\mathrm{NaOH}$ (aq.) $10 \%$ w/w & 23 & 1.9 & 16 & 461 & 1064 & 87 \\
\hline 14 & $\mathrm{NaOH}$ (aq.) $20 \% \mathrm{w} / \mathrm{w}$ & 23 & 2.2 & 12 & 495 & 1172 & 79 \\
\hline 15 & $\mathrm{NaOH}$ (aq.) $10 \% \mathrm{w} / \mathrm{w}+$ Urea $2.5 \%$ w/w & 23 & 2.1 & 14 & 511 & 1224 & 75 \\
\hline 16 & $\mathrm{NaOH}$ (aq.) $20 \% \mathrm{w} / \mathrm{w}+$ Urea $2.5 \% \mathrm{w} / \mathrm{w}$ & 23 & 2.3 & 11 & 475 & 1108 & 69 \\
\hline 17 & $\mathrm{NaOH}$ (aq.) $10 \%$ w/w $+\mathrm{FeT} 2.5 \%$ w/w & 23 & 1.9 & 11 & 422 & 943 & 82 \\
\hline 18 & $\mathrm{NaOH}$ (aq.) $20 \% \mathrm{w} / \mathrm{w}+\mathrm{FeT} 2.5 \% \mathrm{w} / \mathrm{w}$ & 23 & 1.9 & 10 & 367 & 780 & 77 \\
\hline 19 & $\mathrm{FeT} 10 \%$ w/w in $\mathrm{NaOH}$ (aq.) $3 \%$ w/w & 23 & 1.5 & $<10$ & 309 & 617 & 79 \\
\hline 20 & $\mathrm{FeT} 10 \%$ w/w in $\mathrm{NaOH}$ (aq.) $6 \%$ w/w & 23 & 1.1 & $<10$ & 231 & 416 & 70 \\
\hline 21 & $\mathrm{NH}_{4} \mathrm{SCN}$ (aq.) $45 \% \mathrm{w} / \mathrm{w}$ & 30 & 1.6 & 18 & 465 & 1077 & 98 \\
\hline 22 & $\mathrm{NH}_{4} \mathrm{SCN}$ (aq.) $45 \% \mathrm{w} / \mathrm{w}$ & 60 & 1.8 & 19 & 476 & 1111 & 89 \\
\hline 23 & $\begin{array}{l}\mathrm{NH}_{4} \mathrm{SCN} \text { (aq.) } 45 \% \text { w/w }+ \text { Urea } 2.5 \% \\
\text { w/w }\end{array}$ & 30 & 1.7 & 18 & 488 & 1150 & 96 \\
\hline 24 & $\begin{array}{l}\mathrm{NH}_{4} \mathrm{SCN} \text { (aq.) } 45 \% \text { w/w + Urea } 2.5 \% \\
\text { w/w }\end{array}$ & 60 & 1.8 & 17 & 520 & 1253 & 82 \\
\hline 25 & $\mathrm{NH}_{4} \mathrm{SCN} 45 \%$ w/w in $\mathrm{NH}_{3}$ (aq.) $25 \%$ w/w & 30 & 1.9 & 18 & 506 & 1208 & 84 \\
\hline 26 & $\mathrm{NH}_{4} \mathrm{SCN} 60 \%$ w/w in $\mathrm{NH}_{3}$ (aq.) $25 \%$ w/w & 30 & 2.1 & 18 & 517 & 1244 & 85 \\
\hline
\end{tabular}

calculated with Eq. 2. The $\mathrm{CI}$ for $\mathrm{H}_{3} \mathrm{PO}_{4} 80 \%$ could be overestimated since the peak assigned to the (110) reflection of cellulose II may be actually due to the presence of cellulose phosphate.

FTIR spectra in Fig. 4 are presented mainly to check whether or not pretreatments were derivatizing. Except for the cationized sample, the shape of all spectra resembles native cellulose. The absorbance at $897 \mathrm{~cm}^{-1}$ (a) can be assigned to $\mathrm{C} 1-\mathrm{H}$ bending and is sensitive to the supramolecular order of the polymer. The most prominent peak of cellulose is found at $1040 \mathrm{~cm}^{-1}$ (b), related to $\mathrm{C}-\mathrm{O}-\mathrm{C}$ stretching, and it is possible to distinguish multiple peaks at bands assigned to different vibrations of the $\mathrm{C}-\mathrm{C}$ bonds (c). The band at $1623 \mathrm{~cm}^{-1}$ (d) is related to the $\mathrm{O}-\mathrm{H}$ bonds in absorbed water. The peaks found at $2282 \mathrm{~cm}^{-1}$ (e) and $3300 \mathrm{~cm}^{-1}$ (f) can be associated to stretching of $\mathrm{C}-\mathrm{H}$ and $\mathrm{O}-\mathrm{H}$ bonds in cellulose, respectively. Besides these ones, the spectrum of cationic cellulose has notorious peaks at 1427 and $1390 \mathrm{~cm}^{-1}(\mathrm{~g})$, due to the quaternary ammonium group (Aguado et al. 2017).

Influence of the pretreatments on cationization

Table 2, which contains the charge density (CD) and the DS of all samples, is an indication of the reactivity 

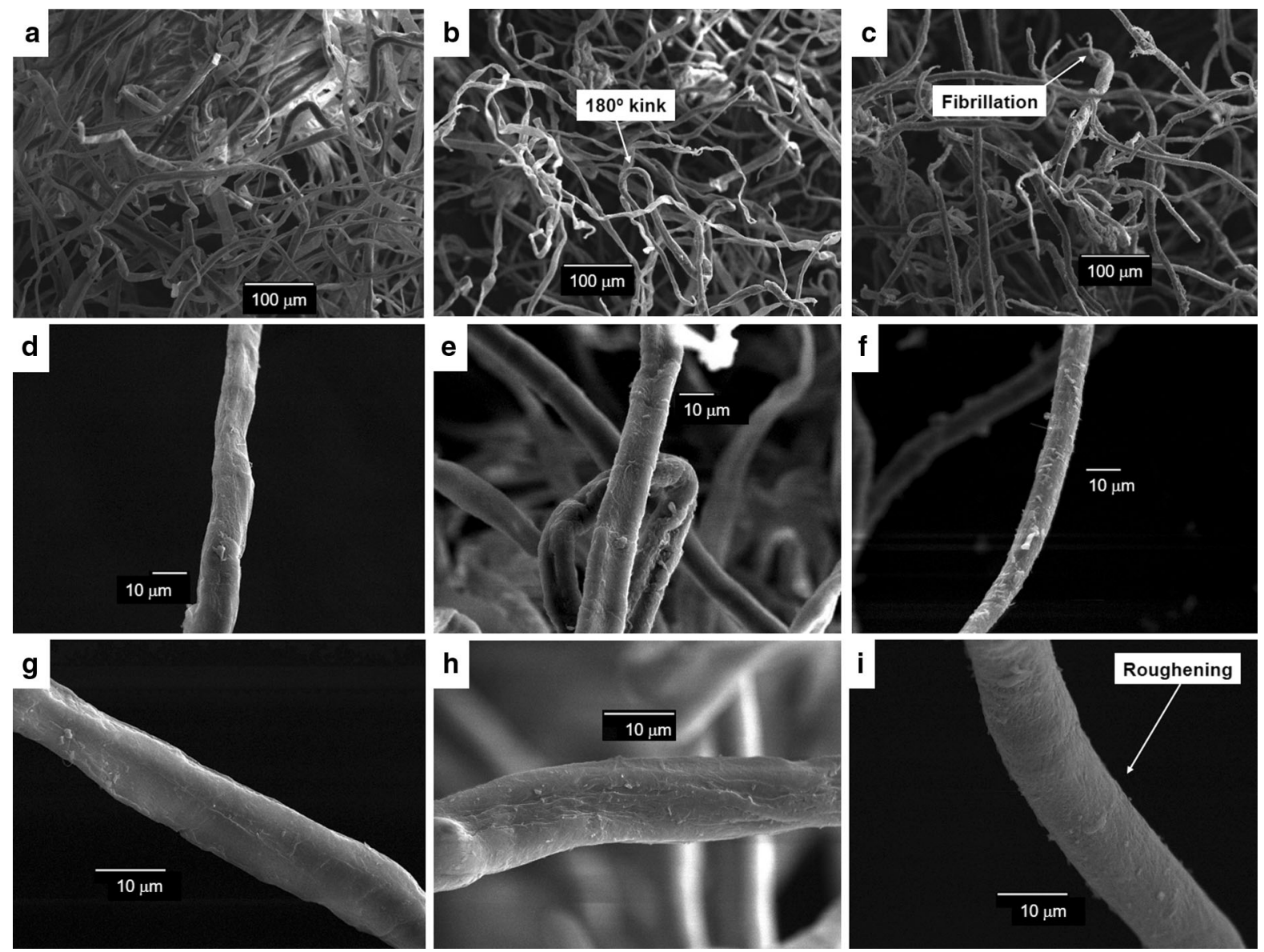

Fig. 2 Micrographs at $\times 200$ of pulp (a), pulp treated with $\mathrm{H}_{3} \mathrm{PO}_{4}(\mathbf{b})$, and pulp treated with $\mathrm{NaOH}(\mathbf{c})$. Micrographs at $\times 1000$ of pulp $(\mathbf{d})$, pulp treated with $\mathrm{SCN}^{-}(\mathbf{e})$, and pulp treated

provided by each pretreatment. If there is a discrepancy between DS and CD, the latter is preferred to indicate the extent of cationization, because the former may carry overestimation due to the presence of cationized hemicellulose or underestimation due to heavier atoms (iron, phosphorus).

A scan at visible light wavelengths was motivated by the observation of yellowing in $\mathrm{NaOH}$-treated samples, and it was confirmed that the absorbance of blue light $(\sim 400-500 \mathrm{~nm})$ increased. To show that this yellowing is not a necessary consequence of cationization, the absorbance values at $457 \mathrm{~nm}$ are displayed for other samples in Table 3. Since absorbance could increase by simply using thicker samples, it should be noted that this information is only valuable when compared with the original pulp. with $\mathrm{NaOH}$ and urea (f). Micrographs at $\times 200$ of pulp (g), pulp treated with $\mathrm{SCN}^{-}$and $\mathrm{NH}_{3}(\mathbf{h})$, and pulp treated with $\mathrm{FeTNa}(\mathbf{i})$

Table 3 also contains the WRV, the $\eta$ and the Schopper-Riegler number of selected cationic samples. The increase in viscosity is not only due to the increase in the molecular mass by the attachment of quaternary ammonium groups, but also to the change in the polymer conformation ( $a$ in Eq. 1) (Harding 1997).

\section{Discussion}

Orthophosphoric acid treatments

A mild swelling agent only affects the amorphous portion of the fiber. Only with strong swelling agents, like $\mathrm{H}_{3} \mathrm{PO}_{4} 80 \%$, the crystalline structure of the fiber was disrupted. This was the only concentration that 
Fig. 3 X-ray diffraction patterns of representative treatments, indicating the Miller indices of the most important planes and the CI (calculated by the area method) at the right side of each spectrum

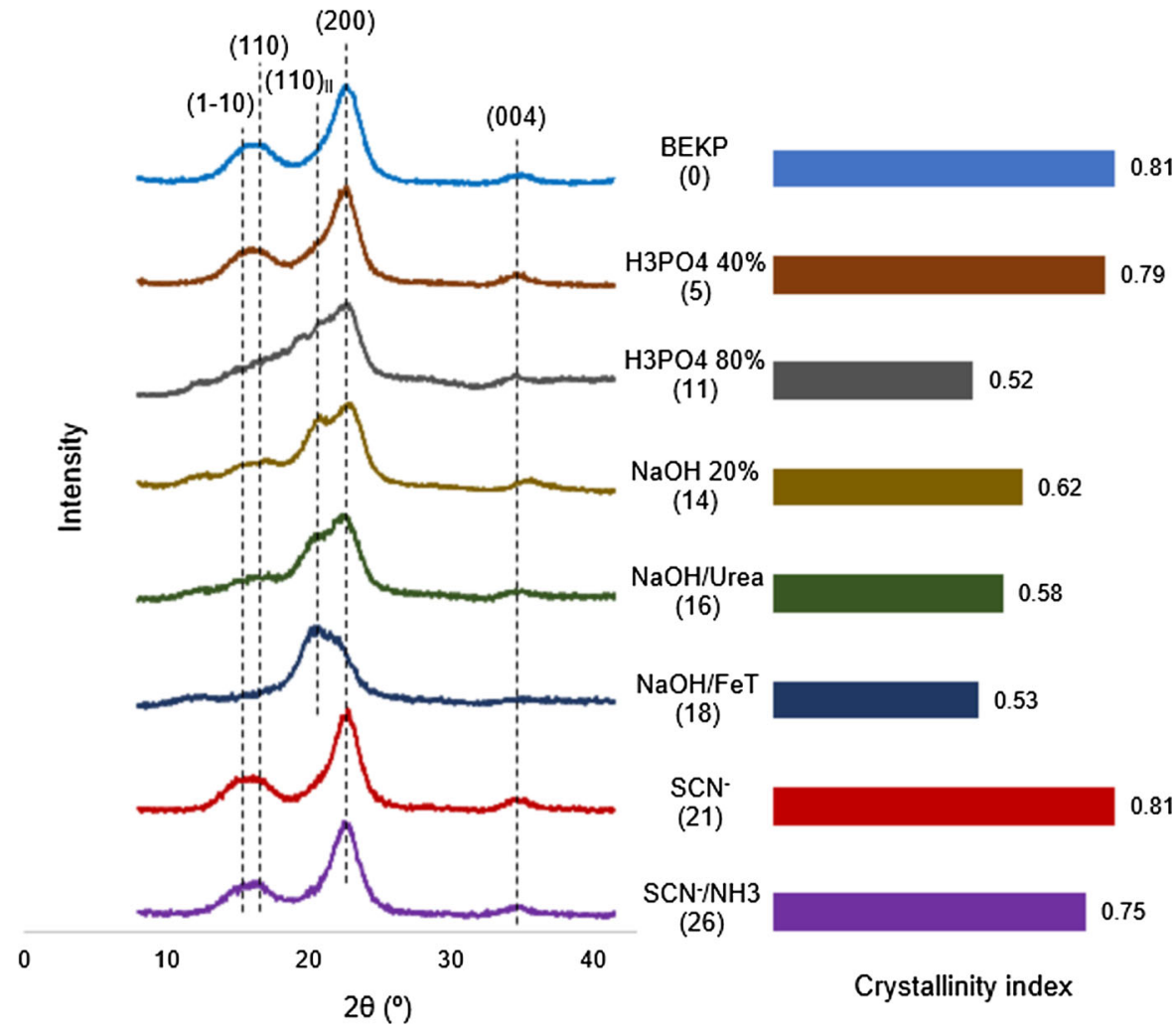

activated cellulose towards high DS and CD values (Table 2). Figure 5 relates those variables to decrystallization.

A mechanism for the phosphate decrystallization of cellulose was proposed by Zhang et al. (2009): first, cellulose is esterified towards cellulose phosphate. This reaction is reversible. Then, water molecules compete with hydroxyl groups from cellulose to form hydrogen bonds with other cellulose chains. As a byreaction, hydrolysis of $\beta$-glycosidic bonds occurs, particularly at high temperature. If it stays aqueous and below $100{ }^{\circ} \mathrm{C}$, cellulose phosphate is easily converted back to free phosphoric acid and amorphous cellulose.

The by-reaction aforementioned, cellulose depolymerization, always happens when treated with any strong mineral acid or even with concentrated weak acids. If conditions are too mild to hydrolyze the crystalline regions, only the amorphous fraction is attacked and solubilized, resulting in chains of highlycrystalline cellulose (Wei et al. 1996). The particularity of phosphoric acid is that it decreases the CI of cellulose. At the same time, it is a decrystallization agent and a depolymerization agent.

In fact, only the pretreatments involving $\mathrm{H}_{3} \mathrm{PO}_{4}$ $60 \%$ or $80 \%$ (Table 1, codes $7-12$ ) led to significant depolymerization of cellulose. The low yields obtained with $\mathrm{H}_{3} \mathrm{PO}_{4} 40 \%$ may be due to the hydrolysis and solubilization of hemicellulose. As for the temperature effect, an increase of $30{ }^{\circ} \mathrm{C}$ led to a decrease in the DP of ca. 500 (samples 8 and 12 with $\mathrm{H}_{3} \mathrm{PO}_{4} 80 \%$ ). As a consequence of high temperatures and high acid concentrations, fiber-fiber interactions were much affected and the Schopper-Riegler number could not even be quantified.

The addition of urea showed little effect on activation, unlike in the pretreatments with $\mathrm{NaOH}$ or thiocyanate. Even though urea has been reported to have a positive influence on phosphorylation, it also inhibits the inverse reaction towards regenerated phosphoric acid and amorphous cellulose (Ghanadpour et al. 2015).

Diffraction patterns in Fig. 3 also support the notion that phosphorylation was reversed in the aqueous media when $\mathrm{H}_{3} \mathrm{PO}_{4}$ concentration was $40 \%$, 


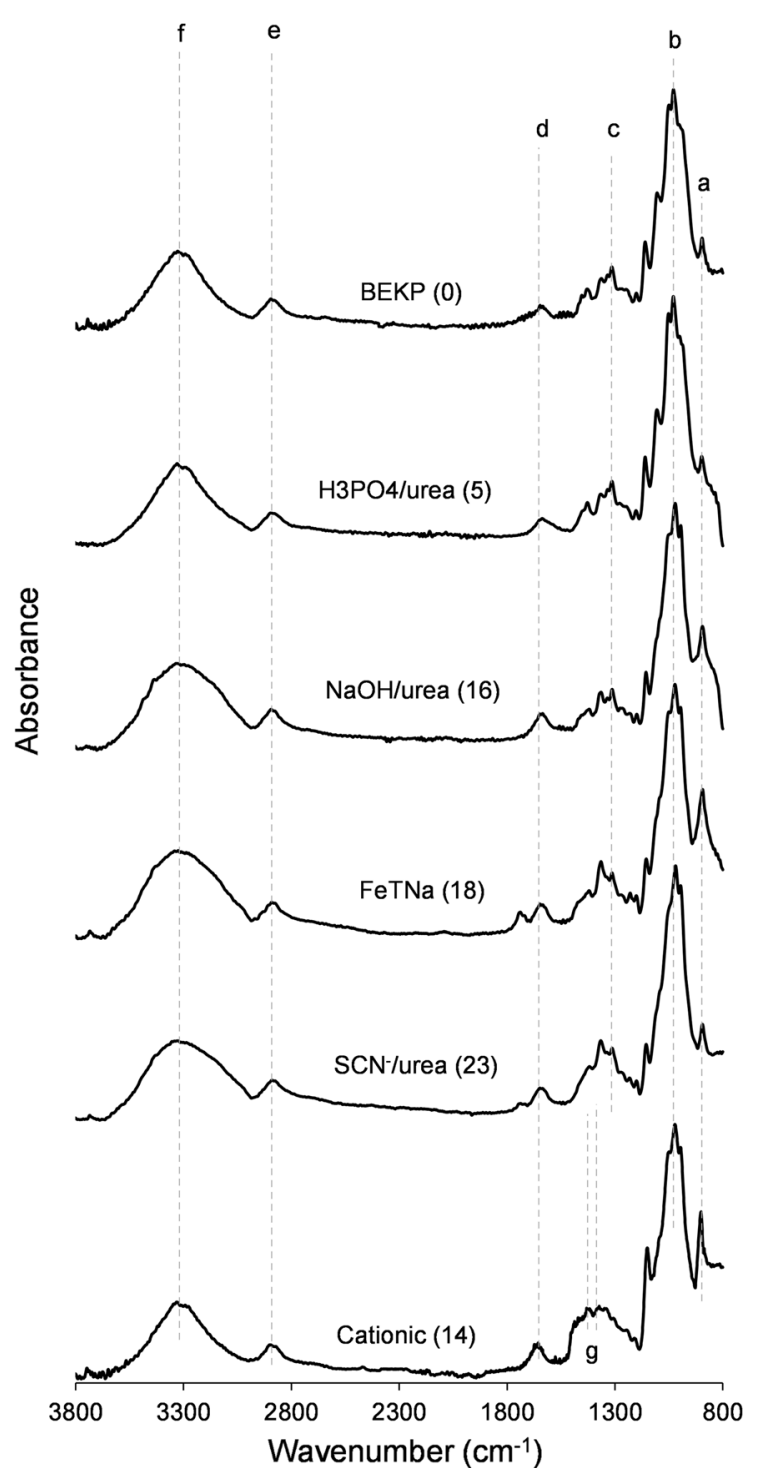

Fig. 4 FTIR spectra of the original pulp, the pulp after some representative pretreatments, and the pulp after a $\mathrm{NaOH} /$ urea treatment followed by cationization

but the shape of the pattern for $\mathrm{H}_{3} \mathrm{PO}_{4} 80 \%$ between $17^{\circ}$ and $20^{\circ}$ resembles that of lowly-substituted cellulose phosphate (Wan Daud et al. 2011). This retention of some phosphate groups is undesirable because of its negative $\mathrm{CD}$.

\section{$\mathrm{NaOH}$ and $\mathrm{NaOH} / \mathrm{urea}$ treatments}

The most obvious effect undergone by fibers in aqueous, strongly alkaline solutions was swelling. Swelling produces internal stresses that, in these cases,
Table 2 Cationicity of the 27 cationized samples, including the original pulp (0) and the 26 pretreated pulps

\begin{tabular}{|c|c|c|}
\hline Code & DS & $\mathrm{CD}(\mathrm{meq} / \mathrm{g})$ \\
\hline 0 & 0.020 & 0.081 \\
\hline 1 & 0.025 & 0.084 \\
\hline 2 & 0.029 & 0.126 \\
\hline 3 & 0.030 & 0.150 \\
\hline 4 & 0.036 & 0.164 \\
\hline 5 & 0.032 & 0.142 \\
\hline 6 & 0.035 & 0.163 \\
\hline 7 & 0.032 & 0.185 \\
\hline 8 & 0.036 & 0.218 \\
\hline 9 & 0.030 & 0.205 \\
\hline 10 & 0.037 & 0.218 \\
\hline 11 & $0.084^{\mathrm{a}}$ & 0.340 \\
\hline 12 & $0.057^{\mathrm{a}}$ & 0.277 \\
\hline 13 & 0.082 & 0.283 \\
\hline 14 & 0.099 & 0.355 \\
\hline 15 & 0.089 & 0.286 \\
\hline 16 & 0.101 & 0.391 \\
\hline 17 & $0.050^{\mathrm{a}}$ & 0.242 \\
\hline 18 & $0.053^{\mathrm{a}}$ & 0.363 \\
\hline 19 & $0.037^{\mathrm{a}}$ & 0.355 \\
\hline 20 & $0.065^{\mathrm{a}}$ & 0.382 \\
\hline 21 & 0.024 & 0.111 \\
\hline 22 & 0.029 & 0.156 \\
\hline 23 & 0.030 & 0.154 \\
\hline 24 & 0.030 & 0.168 \\
\hline 25 & 0.052 & 0.290 \\
\hline 26 & 0.059 & 0.284 \\
\hline
\end{tabular}

${ }^{\text {a} P o s s i b l y ~ u n d e r e s t i m a t e d ~}$

break intermolecular bonds irreversibly, and thus the degree of supramolecular order decreases (Fig. 3). Each fiber became able to retain more water, as shown by WRV (Table 1) and by the peak associated to absorbed water in FTIR spectra (Fig. 4b, d). At the same time, the surface area of the fiber increased, as external fibrillation is clear in Fig 2c, $\mathrm{f}$.

The use of $\mathrm{NaOH}$ to increase reactivity of cellulose is already well-known (Okano and Sarco 1985). This conventional method proved better for cationization (Table 2) than any of the non-alkaline pretreatments. By adding urea, decrystallization increased (Figs. 3 and 5), fibers swelled more, and even higher DS and $\mathrm{CD}$ values were reached by functionalization. Nonetheless, Jin et al. (2007) showed that an aqueous 
Table 3 Properties of certain cationized samples, compared to those of the original pulp

\begin{tabular}{lllll}
\hline Code & WRV $(\mathrm{g} / \mathrm{g})$ & Schopper-Riegler $\left({ }^{\circ} \mathrm{SR}\right)$ & $\eta(\mathrm{mL} / \mathrm{g})$ & $\mathrm{A}_{457}$ \\
\hline 0 (before cationization) & 1.6 & 18 & 491 & 0.134 \\
0 & 1.9 & 18 & 510 & 0.135 \\
5 & 1.9 & 16 & 504 & 0.132 \\
14 & 2.8 & 15 & 617 & 0.144 \\
16 & 3.1 & 14 & 592 & 0.149 \\
18 & 2.8 & 14 & 464 & 0.230 \\
21 & 1.9 & 18 & 496 & 0.136 \\
26 & 2.4 & 19 & 560 & 0.139 \\
\hline
\end{tabular}

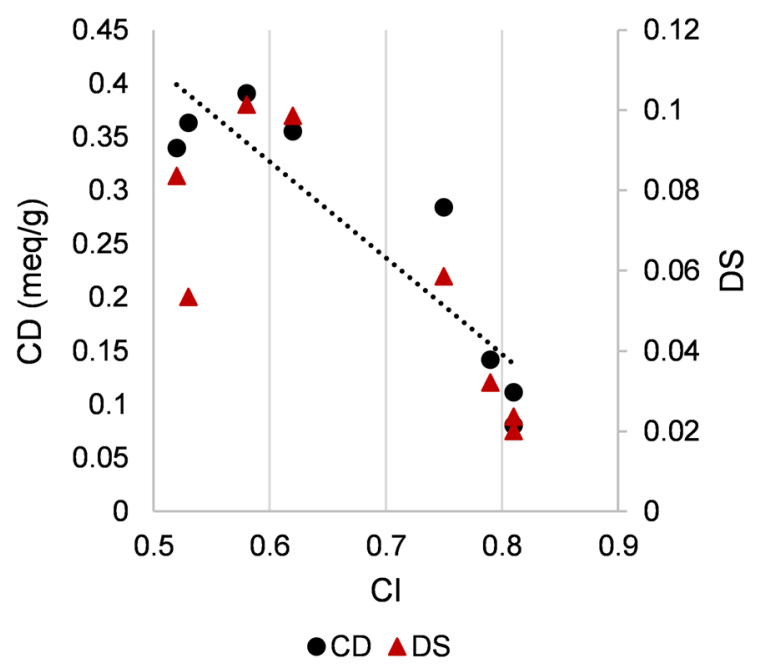

Fig. 5 Influence of the extent of the decrystallization on the reactivity towards cationization, expressed as the degree of substitution (DS) and as the charge density (CD)

solution of urea alone barely amorphizes cellulose. The role of urea in cellulose dissolution is still not clear and different explanations have been suggested, e.g., that urea hydrates become bonded to the $\mathrm{NaOH}$ hydrates complexed with cellulose, as many studies show no direct interaction between urea and cellulose (Budtova and Navard 2016).

However, these alkaline pretreatments have important drawbacks if these functionalized fibers are meant to be used to form sheets of acceptable strength. Upon treatment with $\mathrm{NaOH}$ or $\mathrm{NaOH} /$ urea, cellulose $\mathrm{I} \beta$ was partially converted into amorphous cellulose and into cellulose II (Fig. 3). Cellulose II keeps the fiber structure, but it is mechanically inferior to cellulose I $\beta$. The low Schopper-Riegler numbers, particularly in contrast to the high WRV, show that inter-fiber water is easily removed by fast filtration. These weakening ways to activate cellulose are undoubtedly useful when aiming at soluble derivatives, but not for applications in which strength is required. Drainability improved after cationization (Table 3), but SR numbers are still far from the original pulp and this would hinder sheet forming.

Yellowing of cationized samples was another undesired side-effect (Table 3). While this was only caused by cationization following alkaline pretreatments, the pretreatments themselves (at room temperature) did not produce any change in color. However, decrystallization likely made cellulose more sensitive to heat-induced oxidation during cationization. In fact, it has been reported that etherifying nanocellulose with quaternary ammonium groups at $120{ }^{\circ} \mathrm{C}$ results in a brown product (Ho et al. 2011).

Treatments with FeTNa

It has been verified that samples treated with $\mathrm{H}_{3} \mathrm{PO}_{4}$ 20-60\% did not retain phosphate groups, no carbamate groups were appreciated in the FTIR spectra of urea-treated samples (Fig. 4), and cellulose was not esterified towards cellulose thiocyanate with $\mathrm{NH}_{4} \mathrm{SCN}$ (Figs. 3 and 4). However, regardless the concentration, $\mathrm{Fe}(\mathrm{III})$ remained bound to cellulose after dilution, neutralization, and rinsing with distilled water, hot water and different organic solvents. Such binding was not expected, as in previous works FeTNa is regarded as a non-derivatizing solvent system (El-Wakil and Hassan 2008), although the complex was both prepared and applied in different ways.

It can be proposed that, in the first place, cellulose undergoes swelling and activated in the alkaline medium, being then attacked by FeT. The dipolar bonds between the central $\mathrm{Fe}$ atom and the $-\mathrm{OH}$ of tartaric acid groups are replaced by dipolar bonds between $\mathrm{Fe}$ and the different $-\mathrm{OH}$ groups of 
anhydroglucose units. Unlike urea, FeTNa interacts with cellulose in terms of chemical bonds, but this interaction was not expected to be stable. Besides binding to cellulose and making it less crystalline, the attack by the iron complex promotes the switch from cellulose I to cellulose II (Figs. 3 and 4). In fact, the lack of cellulose I, a case in which the peak for the (200) plane simply becomes unappreciable, was observed in only one of the X-ray diffraction patterns, corresponding to $20 \% \mathrm{NaOH}$ and only $2.5 \% \mathrm{FeT}$.

During the treatments with FeT 10\% (Table 1, codes 19 and 20), fibers were rapidly attacked and disintegrated. The harsh decrease in pulp viscosity (Table 1) is not only due to depolymerization, which undoubtedly happened to a lesser extent, but also to the new conformation of polymer chains when fibers were regenerated (lower $M$ and $a$ parameters of Eq. 1).

Their capability to retain inter-fiber water is very low, as indicated by the Schopper-Riegler number (Table 1). Due to their weakness, these fibers are not suitable for papermaking. Nonetheless, a high charge density was obtained after cationization (Table 2), which may be useful for other applications.

Ammonium thiocyanate treatments

$\mathrm{NH}_{4} \mathrm{SCN}$ did not swell wood pulp fibers to a high degree and, as cellulose activator, its performance was clearly worse than that of $\mathrm{NaOH}$ and 40-60\% phosphoric acid. Accordingly, the structural changes inflicted to cellulose were very slight, with no appreciable depolymerization (Table 1) and little decrystallization (Figs. 3 and 4), but fibers preserved their strength.

The big size of the $\mathrm{NH}_{4}{ }^{+}$ion in the aqueous solutions of $\mathrm{NH}_{4} \mathrm{SCN}$ was probably the main cause for these drawbacks. The influence of the cation has been explained by Mahmud-Ali and Bechtold (2015), who achieved high degrees of fiber swelling with NaSCN/ urea. According to them, replacing some water molecules by urea in this complex system resulted in better activation, which was expected because the latter can prevent cellulose chains from aggregation by steric effects.

In contrast to aqueous systems, it is known that cold solutions of $\mathrm{NH}_{4} \mathrm{SCN}$ in anhydrous ammonia can even dissolve high-DP cellulose fibers (Hudson et al. 1983). In this work, aqueous $\mathrm{NH}_{3}$ was used to avoid dissolution. The presence of $\mathrm{NH}_{3}$ molecules eased the dissolution of $\mathrm{NH}_{4} \mathrm{SCN}$ in the medium by increasing the saturation concentration, caused fiber swelling by themselves, and partially replaced water in the complex bonding systems. As a result, cationic fibers that had undergone these treatments (Table 1, codes 25 and 26) achieved values of substitution and charge density in the medium range (Table 2), but keeping most cellulose I, high DP, low yellowness and high Schopper-Riegler numbers (Table 3). The viscosity values obtained (Table 1), higher than that of the original pulp $(491 \mathrm{~mL} / \mathrm{g})$, mean that the chemical agent could have selectively dissolved hemicellulose or low-DP cellulose.

\section{Conclusions}

- Bleached wood pulp was etherified with (3-chloro2-hydroxypropyl)trimethylammonium chloride, attaining degrees of substitution as low as 0.02 when no pretreatment was applied.

- Alkalization with $\mathrm{NaOH}$ was used to activate cellulose, resulting in relatively high degrees of substitution (0.1) and charge density values once cationized. Besides, the addition of urea enhanced the effect of $\mathrm{NaOH}$, namely swelling, dissolution, and decrystallization. However, fibers became less capable of retaining inter-fiber water upon sheet forming, and also less white when cationized.

- An iron(III)-tartrate complex promoted both decrystallization and conversion of cellulose I into cellulose II, but the fibers were severely weakened.

- Alternative pretreatments consisting on orthophosphoric acid and ammonium thiocyanate were tested. It was found that the former needed a high concentration (80\%) to activate cellulose towards degrees of substitution above 0.05 , and fibers were unusable for papermaking due to the severe treatments. Ammonium thiocyanate was ineffective unless mixed with aqueous ammonia, which resulted in good fibers activation while keeping good drainability.

Acknowledgments Roberto Aguado is thankful to Asociación Universitaria Iberoamericana de Posgrado for the grant to fund an internship in Coimbra. Ana F. Lourenço acknowledges Fundação para a Ciência e Tecnologia for the Ph.D. Grant SFRH/BDE/108095/2015. 


\section{References}

Aguado R, Lourenço AF, Ferreira PJ, Moral A, Tijero A (2017) Cationic cellulosic derivatives as flocculants in papermaking. Cellulose 24:3015-3027

Aguado R, Moral A, Tijero A (2018) Cationic fibers from lignocellulosic residues: making waste more appealing for papermaking. J Clean Prod 174:1503-1512

Budtova T, Navard P (2016) Cellulose in $\mathrm{NaOH}-$ water based solvents: a review. Cellulose 23:5-55

Eckelt J, Knopf A, Röder T, Weber HK, Sixta H, Wolf BA (2011) Viscosity-molecular weight relationship for cellulose solutions in either NMMO monohydrate or cuen. J Appl Polym Sci 119:670-676

El-Wakil NA, Hassan ML (2008) Structural changes of regenerated cellulose dissolved in FeTNa, $\mathrm{NaOH} /$ thiourea, and NMMO systems. J Appl Polym Sci 109:2862-2871

Fischer S (2004) Unconventional dissolution and derivatization of cellulose. Lenzinger Ber 83:71-78

French AD (2014) Idealized powder diffraction patterns for cellulose polymorphs. Cellulose 21:885-896

Ghanadpour M, Carosio F, Larsson PT, Wågberg L (2015) Phosphorylated cellulose nanofibrils: a renewable nanomaterial for the preparation of intrinsically flame-retardant materials. Biomacromolecules 16:3399-3410

Gruber E, Granzow C, Ott T (1996) Neue Wege zur Kationisierung von Cellulose. Das Papier 50:729-734

Harding SE (1997) The intrinsic viscosity of biological macromolecules. Progress in measurement, interpretation and application to structure in dilute solution. Prog Biophys Mol Biol 68:207-262

Hedlund A, Germgård U (2007) Some aspects on the kinetics of etherification in the preparation of CMC. Cellulose 14:161-169

Heinze T (2004) Chemical functionalization of cellulose. In: Dumitriu S (ed) Polysaccharides: structural diversity and functional versatility, 2nd edn. CRC Press, New York, pp 551-5907

Ho TTT, Zimmermann T, Hauert R, Caseri W (2011) Preparation and characterization of cationic nanofibrillated cellulose from etherification and high-shear disintegration processes. Cellulose 18:1391-1406

Hudson SM, Cuculo JA, Wadsworth LC (1983) The solubility of cellulose in liquid ammonia/ammonium thiocyanate solution: the effect of composition and temperature on dissolution and solution properties. Polym Chem 21:651-670

Jin H, Zha C, Gu L (2007) Direct dissolution of cellulose in $\mathrm{NaOH} /$ thiourea/urea aqueous solution. Carbohydr Res 342:851-858

Littunen K, Snoei de Castro J, Samoylenko A, Xu Q, Quaggin S, Vainio S, Seppälä J (2016) Synthesis of cationized nanofibrillated cellulose and its antimicrobial properties. Eur Polym J 75:116-124
Mahmud-Ali A, Bechtold T (2015) Aqueous thiocyanate-urea solution as a powerful non-alkaline swelling agent for cellulose fibres. Carbohydr Polym 116:124-130

Mayr M, Odabas N, Eckhart R, Henniges U, Bauer W (2017) Cationization of lignocellulose as a means to enhance paper strength properties. BioResources 12:9338-9347

Montplaisir D, Chabot B, Daneault C (2006) Cationisation of thermomechanical pulp fibres. Part 2: influence of strength and retention. Pulp Pap Canada 117:39-42

Nishiyama Y, Langan P, Chanzy H (2002) Crystal structure and hydrogen-bonding system in cellulose $\mathrm{I} \beta$ from synchrotron $\mathrm{X}$-ray and neutron fiber diffraction. J Am Chem Soc 124:9074-9082

Okano T, Sarco A (1985) Mercerization of cellulose II: alkalicellulose intermediates and a possible mercerization mechanism. J Appl Polym Sci 30:325-332

Prado HJ, Matulewicz MC (2014) Cationization of polysaccharides: a path to greener derivatives with many industrial applications. Eur Polym J 52:53-75

Sehaqui H, Mautner A, Perez de Larraya U, Pfenninger N, Tingaut P, Zimmermann T (2016) Cationic cellulose nanofibers from waste pulp residues and their nitrate, fluoride, sulphate and phosphate adsorption properties. Carbohydr Polym 135:334-340

Sirviö JA, Anttila AK, Pirttilä AM, Liimatainen H, Kilpeläinen I, Niinimäki J, Hormi O (2014) Cationic wood cellulose films with high strength and bacterial anti-adhesive properties. Cellulose 21:3573-3583

Sood YV, Tyagi R, Tyagi S, Pande PC, Tondon R (2010) Surface charge of different paper making raw materials and its influence on paper properties. J Sci Ind Res 69:300-304

Vinogradov VV, Mizerovskii LN, Akaev OP (2002) Reaction of cellulose with aqueous solutions of orthophosphoric acid. Fibre Chem 34:167-171

Vu Mahn H, Ozturk HB, Bechtold T (2010) Swelling and dissolution mechanism of lyocell fiber in aqueous alkaline solution containing ferric tartaric acid complex. Cellulose 17:521-532

Wan Daud WR, Haafiz MKM, Seeni A (2011) Cellulose phosphate from oil palm biomass as potential biomaterials. BioResources 6:1719-1740

Wei S, Kumar V, Banker GS (1996) Phosphoric acid mediated depolymerization and decrystallization of cellulose: preparation of low crystallinity cellulose a new pharmaceutical excipient. Int J Pharm 142:175-181

Zhang J, Zhang J, Lin L, Chen T, Zhang J, Liu S, Li Z, Ouyang P (2009) Dissolution of microcrystalline cellulose in phosphoric acid-molecular changes and kinetics. Molecules 14:5027-5041

Publisher's Note Springer Nature remains neutral with regard to jurisdictional claims in published maps and institutional affiliations. 